\title{
Thermodiffusion problem of determining the stress state of a steel shell structure
}

\author{
Igor Emelyanov 1, 2,* \\ ${ }^{1}$ Ural State University of Railway Transport, Kolmogorova st., 66, Ekaterinburg, 620034, Russia \\ ${ }^{2}$ The Institute of Engineering Science RAS (Ural Branch), Tekhnicheskaya str., 18b, Ekaterinburg, \\ 620049 Russia
}

\begin{abstract}
In various engineering industries, including railroad transport, metal structural elements are sometimes operated under external thermomechanical loads while being also exposed to aggressive environments. The impact of an aggressive environment on the mechanical properties of metals is one of the dominant factors, which determines structure strength and service life, as it usually deteriorates the mechanical properties of materials. The thermodiffusion problem of determining the stress state of a steel shell structure is considered. The subject of the study is a compound steel shell of revolution loaded with internal pressure and operating in a hydrogen-containing environment at high temperatures. The purpose of this study is to determine the stress state of the shell taking into account the changes in the mechanical properties due to simultaneous exposure to temperature and hydrogen. In the suggested approach, the thermodiffusion and mechanical problems are coupled by taking into account the changes in stress-strain diagrams of steel samples as temperature and hydrogen concentration increase. The boundary problem of heat conduction and diffusion is solved using the finite elements method. The system of differential equations for the boundary problem of shell stress state is integrated using S. K. Godunov's method of discrete orthogonalization. The method is based on stepwise orthogonalization of the solution vectors of the Cauchy problem. The obtained solution allowed determining the site of maximal stresses and drawing conclusions on the strength of the studied structure when increasing hydrogen concentration and internal pressure.
\end{abstract}

\section{Introduction}

The shell steel structural elements are widely used as structural elements in various industrial sectors, including railroad transport. Such structural elements are sometimes not only operated under external mechanical loads, but are also exposed to heating and aggressive environments. The impact of an aggressive environment, including a hydrogencontaining one, on the mechanical properties of metals when operating structures is one of

\footnotetext{
* Corresponding author: emelyanov@imach.uran.ru
} 
the dominant but poorly studied factors, which determines the strength and service life of many potentially hazardous objects $[1,2]$.

The changes in the mechanical properties of steels when exposed to a hydrogencontaining environment have been studied for several decades due to the large impact such processes exert on the strength and service life of various structures, e.g., main pipelines. Two directions can be distinguished in the investigation of the problem of hydrogeninduced degradation. The first one deals with a further study of the fundamentals and the processes of hydrogen embrittlement and ageing of metals, while the second focuses on the development of models and methods for predicting changes in the stress state to evaluate the remaining service life of the structure.

It is known that the danger of hydrogen's impact on metal lies in the fact that this process takes place inside the metal and does now show any external signs. As changes in the mechanical properties cannot be diagnosed with any means, to evaluate the strength of various structures operated in the hydrogen-containing environments, mathematical models based on the available experimental data on hydrogen's impact on metal need to be developed.

Previous research devoted to the problem of hydrogen's interaction with metals and alloys includes hundreds of studies. We shall only take modern papers in [3-5] that provide a review of the monographs and articles covering numerous aspects of this problem, and, in particular, the changes in the stress state of structures during hydrogenation. Incorporation of hydrogen into various steels can proceed via one of two different mechanisms. The first one takes place as a result of electrochemical processes. This process is referred to as lowtemperature hydrogen embrittlement (usually at temperatures up to $\mathrm{T}=200{ }^{\circ} \mathrm{C}$ ). The second one occurs as a result of thermal dissociation of hydrogen molecules with atomic hydrogen produced. This process is commonly referred to as high-temperature hydrogeninduced corrosion, as it usually takes place at elevated temperatures $T$ and pressures $p$. Upon simultaneous exposure to high temperatures and pressures, a physical and chemical interaction of hydrogen with metal takes place, leading also to irreversible changes in the initial mechanical properties. The mechanism of hydrogen-induced corrosion is based on the interaction of hydrogen with carbon, which produces methane. This reaction starts from the surface, leading to carbon depletion and formation of microcracks that gradually propagate through the metal, deteriorating its strength and plastic properties.

Hydrogen's impact on steel results in changes in its stress-strain diagram. The connection between stress $\sigma$, deformation $\varepsilon$, and hydrogen concentration $c$ in the thermodiffusion problem of determination of the stress state of a structural element when the latter is operated in a hydrogen-containing environment can be represented as $\sigma=f(\varepsilon, c$, $T$ ). Therefore, determination of the stress state of structural elements calls for application of both current methods of computational mechanics and experimental methods to study the changes in the properties of materials exposed to aggressive environments. Thus, when solving the problems of determining the strength of steel structural elements in contact with hydrogen, it is necessary to take into account the phenomenological effects of the development of deformations in hydrogen-containing media. The phenomenological approach will make it possible to most simply establish some general relationships between the parameters characterizing the strength of the structural element during operation. However, when solving applied problems, from the whole variety of experimental data obtained for various steels, it is not so easy to select data that fully meets the necessary requirements for determining the strength and durability of structural elements. This is caused by the great variety of experimental methods, as well as the fact that experimental information is presented in such a way that it is not always possible for mechanics to use it. 


\section{Statement of the Problem and Solutions (Equations)}

The paper provides an approach to solving the thermodiffusion problem of determination of the stress state of a steel shell structure operated in an aggressive environment. The structure is assumed to be a thin-walled shell of revolution with thickness $h$, with varying geometric and mechanical parameters along the generatrix. Let us refer the shell to a continuous median surface with orthogonal curvilinear coordinates $\mathrm{S}, \theta, \gamma$, where $s$ is meridian and $\theta$ is the circumferential coordinate, $\gamma$ is the direction of external normal to the shell surface (Fig. 1). Therefore, $-h / 2 \leq \gamma \leq h / 2$.

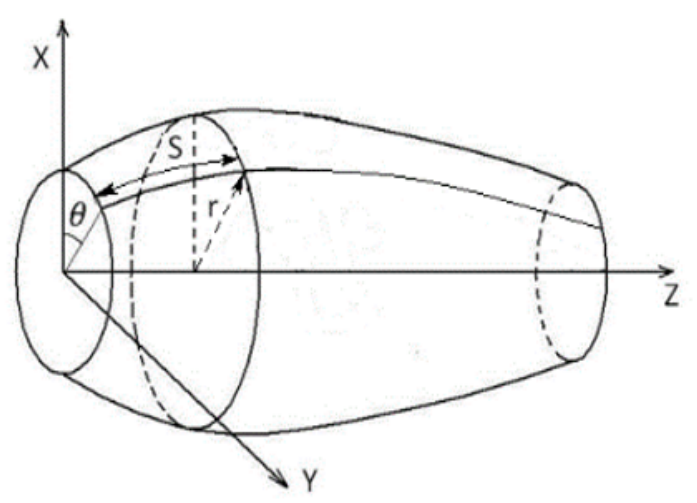

Fig. 1. Shell of revolution in a Cartesian coordinate system.

The inner shell surface comes into contact with an aggressive hydrogen-containing environment with excess pressure $p$ and temperature $T$, from which hydrogen diffuses into the shell to change the mechanical properties of material in the course of operation. The solving of this coupled thermodiffusion problem can be provided as follows: 1) solving the heat conduction problem to determine the temperature distribution in the shell over time $T(t)$; 2) solving the problem of hydrogen diffusion into metal to determine hydrogen distribution taking heating into account $c(t, T)$; 3) obtaining experimental dependences between stress $\sigma$, deformation $\varepsilon$, hydrogen concentration $c$ and temperature $T$ for samples $\sigma=f(\varepsilon, c, T)$ with uniaxial stress state; 4) determination of the stress state of the studied structure taking into account the changed physical and chemical properties of the material $\sigma=f(p, c, T)$ at a given time $t$.

Changes in temperature at any given point of an isotropic body due to heat conductivity will be described by the following equation $[6,7]$ :

$$
\frac{\partial T}{\partial t}=a \Delta(T)+q(x, y, z, T)
$$

$$
\Delta=\nabla^{2}=\frac{\partial^{2}}{\partial x^{2}}+\frac{\partial^{2}}{\partial y^{2}}+\frac{\partial^{2}}{\partial z^{2}} \text { is the Laplace operator, }
$$

$q(x, y, z, T)$ is the heat source and sink function, $a$ is the temperature conductivity coefficient.

The effect of temperature on the shell surface at every given time shall be defined by boundary conditions. Four types of boundary conditions are commonly used in the heat 
conduction problems. In this paper, we shall use conditions of the first kind, specifying the temperature distribution at the shell surface at every given time

$$
T\left(\gamma=-\frac{h}{2}, t\right)=T_{H}
$$

Where $T_{H}$ is the initial temperature at the inner shell surface.

Since the shell is in contact with hydrogen, the latter starts to diffuse into the metal wall. It is known that diffusion equations and heat conduction equations are completely identical $[6,7,8]$. Therefore, the methods for solving diffusion and heat conduction problems are also identical. It then follows that the differential equation for diffusion, similar to the differential equation for heat conduction, can be provided as $[6,7]$

$$
\frac{\partial c}{\partial t}=D \Delta(c)+f(x, y, z, t)
$$

where $f(x, y, z, t)$ is a function describing the creation or disappearance of the diffusing molecules at each point in the medium, $\mathrm{D}$ is the diffusion coefficient.

Bulk concentration of hydrogen in metals is measured as hydrogen content in ppm (1 $\mathrm{cm}^{3} / 100 \mathrm{~g}$ ), and at high pressures and temperatures hydrogen solubility in certain steels can reach 300-400 ppm [9].

The diffusion problem also requires setting boundary conditions at the shell surface. Let us assume that the boundary conditions for this problem (3) are the values of hydrogen concentration that should be known at the shell surface from certain physical considerations. Adopting an assumption of rapid mixing in the hydrogen-containing environment, we may set the boundary conditions of the first kind [6]

$$
c\left(\gamma=-\frac{h}{2}, t\right)=c_{H}
$$

where $c_{H}$ is the initial concentration of hydrogen at the shell inner surface.

An axially symmetric stress-strain state of thin-walled structures, with the assumption of Kirchhoff-Love hypotheses, is described by a system of ordinary sixth-order differential equations $[10,11]$

$$
\frac{d \bar{Y}}{d s}=P_{i j} \bar{Y}+\bar{f}, \quad(i, j=1,2, \ldots 6), \bar{Y}=\left\{N_{r}, N_{z}, M_{s}, u_{r}, u_{z}, \vartheta_{s},\right\}
$$

with boundary conditions

$$
B_{1} \bar{Y}\left(s_{0}\right)=\bar{b}_{1}, B_{2} \bar{Y}\left(s_{L}\right)=\bar{b}_{2}
$$

Where $N_{r}, N_{z}$ are the radial and axial forces; $u_{r}, u_{z}$ are the corresponding displacements; $\mathrm{M}_{\mathrm{s}}$ is meridian bending moment; $\vartheta_{s}$ is a normal rotation angle. Matrix elements $P_{i j}$ are determined by the geometric and mechanical properties of the shell. The column vector of constant terms $\bar{f}$ depends on the surface mechanical loads applied to the shell. $B_{1}$ and $B_{2}$ are known matrices; $\bar{b}_{1}$ and $\bar{b}_{2}$ are known vectors. Matrix elements $P_{i j}, B_{l}, B_{2}$ and $\bar{f}$ are given in the papers [10-13]. 
Thus, the problem of determination of the stress state of a shell structure shall be described by a set of equations from three systems of differential equations (1), (3), and (6) with certain initial and boundary conditions.

\section{Adopted assumptions and research methods}

First, the boundary problem of heat conduction is solved. Taking into account axial symmetry, the equation for nonstationary heat conduction in a cylindrical coordinate system $z, r$ shall be written as [12]

$$
\frac{\partial}{\partial z}\left(\lambda \frac{\partial T}{\partial z}\right)+\frac{1}{r} \frac{\partial}{\partial r}\left(r \lambda \frac{\partial T}{\partial r}\right)=\frac{\lambda}{a} \frac{\partial T}{\partial t}
$$

where $\lambda$ is the thermal conductivity coefficient, $r(s)$ is the shell's median radius (Fig. 1).

When solving problem (1), we use an approach based on substituting the heat conduction equation with an equivalent variational equation [12], which is solved using the finite elements method following the same scheme we would use to solve a heat conduction problem. The computing program was verified by solving the problem of rod heating from its end, which has an exact analytical solution. Thus, after solving equation (7), we can determine the temperature distribution over the shell thickness $T(\gamma, t)$ at any time. For the diffusion problem, we use the computing program for calculation of nonstationary heat conduction. The diffusion coefficient required for calculation characterizes the efficiency of diffusional displacement of the studied compound and is measured in $\mathrm{m}^{2} / \mathrm{s}$, as is the temperature conductivity coefficient $a$ in the problems of heat conduction. The value of the diffusion coefficient for the desired pair diffusing compound-medium is most often determined experimentally. It is known that the velocity of the diffusion process is several orders of magnitude lower than the velocity of heat propagation. Therefore, when solving the diffusion problem, we can assume that a stationary steady-state temperature field exists in the shell at any time. Diffusion strongly depends on the temperature of a body [6], so the diffusion coefficient $D$ will also be temperature-dependent. Thus, after solving the diffusion equation, hydrogen distribution over the shell body $c(\gamma, t, T)$ at any time can be found. The numerical results to the given problem of heat conduction and diffusion for a steel shell structure can be found in the papers $[14,15]$.

To solve the problem of determination of the stress state of the shell, experimental dependences between stress, deformation, concentration, and temperature are needed. Such data can be obtained when testing samples for simple tension at fixed values of $c$ and $T$. To solve the stated problem, let us use the experimental data for steel given in the paper [16]. In this study, the stress-strain curves for a steel sample were approximated with a bilinear curve with yield point $\sigma_{T}, \varepsilon_{T}$ and breaking strength point $\sigma_{B}, \varepsilon_{B}$ for three different hydrogen concentrations (table 1):

Table 1. Hydrogen concentrations.

\begin{tabular}{|c|c|c|c|c|}
\hline$\sigma_{Y}, \mathrm{MPa}$ & $\varepsilon_{Y}$ & $\sigma_{u l t}, \mathrm{MPa}$ & $\varepsilon_{u l t}$ & $\mathrm{C}, \mathrm{ppm}$ \\
\hline 336 & 0.00168 & 514 & 0.2 & 1.68 \\
\hline 372 & 0.00186 & 506 & 0.2 & 9.47 \\
\hline 409 & 0.002045 & 414 & 0.2 & 15.93 \\
\hline
\end{tabular}


The linear boundary problem of shell static (5) is solved using S. K. Godunov's method of discrete orthogonalization [17] that "normalizes" the solution when boundary and local effects arise in the shell.

The quoted mechanical characteristics (table 1) demonstrate that at a given test temperature and with small deformations, these three bilinear curves have one elastic modulus. As hydrogen concentration in the given steel increases, the mechanical properties change differently: the yield point increases, while the breaking strength point decreases. The effect of stress-strain curve variation in the plastic deformations zone is observed. Therefore, under significant external mechanical loads, the problem is physically nonlinear and dependent on hydrogen concentration. Such a physically nonlinear problem will be described by the system of differential equations (5), while the connection between stress and deformation should be linearized using the method of additional strains. This connection is represented by Hooke's law but with additional terms to take into account the dependence of the mechanical properties of material on deformation, temperature and hydrogen concentration. At the same time, the complex stress state of the shell will correspond to a uniaxial state under simple sample tension at $C=$ const $[11,12]$

$$
G=\frac{\sigma}{\sqrt{3}}, H=\frac{1+v}{\sqrt{3}} \varepsilon
$$

where $\sigma$ and $\varepsilon$ are the stress and deformation under simple sample tension, and $v$ is the Poisson's ratio. The intensities of tangential stresses and shear deformations in the shell $G$ and $H$ are determined as

$$
\begin{gathered}
G=\sqrt{(1 / 3) \cdot\left(\sigma_{s}^{2}-\sigma_{s} \sigma_{\vartheta}+\sigma_{\vartheta}^{2}\right)} \\
H=\sqrt{(1 / 6) \cdot\left[\left(\varepsilon_{s}-\varepsilon_{\gamma}\right)^{2}+\left(\varepsilon_{\gamma}-\varepsilon_{\vartheta}\right)^{2}+\left(\varepsilon_{\vartheta}-\varepsilon_{s}\right)^{2}\right]}
\end{gathered}
$$

where $\sigma_{s}$ and $\sigma_{\vartheta}$ are the meridian and circumferential stresses, respectively, and $\varepsilon_{s}, \varepsilon_{\vartheta}, \varepsilon_{\gamma}$ are the deformation components along the meridian, circumference, and normal to the surface of the shell.

\section{Research results}

The thermodiffusion problem of determination of the stress state of a steel shell structure, the casing of a diffusion apparatus, has been solved. This apparatus serves to produce especially pure hydrogen. The casing of the apparatus is approximated with three shell elements: plate, torus and cylinder (Fig. 2). Wall thickness $h=0.018 \mathrm{~m}$, median radius of the cylindrical part $R=0.65 \mathrm{~m}$. The boundary conditions were chosen so as to maximally reflect actual casing operation. The casing is loaded with internal pressure $p=2.5 \mathrm{MPa}$. Operating temperature up to $200 \mathrm{C}^{\circ}$. As a result of prolonged operation of the apparatus, hydrogen diffuses into the casing walls, which leads to changes in the mechanical properties of material and deterioration of its strength [19].

Fig. 3 shows temperature distribution over the wall thickness. Curves $1-5$ correspond to $1,5,10,100,200$ seconds of heating. From the performed calculations it follows that after operating the diffusion apparatus for 4 minutes, an equal temperature is reached along the entire wall thickness. 
Fig. 4 shows hydrogen distribution over the wall thickness in the cylindrical part of the casing. Curves 1-3 correspond to $20 \mathrm{~min}, 1.66$ hours and 33.33 hours of diffusion apparatus operation.

After integrating the system (6), we have stress components ( $\sigma_{s}$ is meridian, $\sigma_{\theta}$ is circumferential) at the outer and inner shell surfaces and displacements of the median surface along two directions $u_{r}, u_{z}$ at each shell point, from which conclusions on the strength of the studied thin-walled structure can be inferred. From the calculation results it follows that for all the adopted mechanical characteristics (9), i.e. for any hydrogen concentration, the shell material works in the elastic region of the diagram. As these bilinear curves have the same elastic modulus ( $\left.E=2 \times 10^{5} \mathrm{MPa}\right)$, the stresses are equal. Thus, for the adopted operational parameters, no residual deformations developed in the material of the protective casing of the diffusion apparatus, and increased hydrogen concentration does not affect the safety margin of the casing.

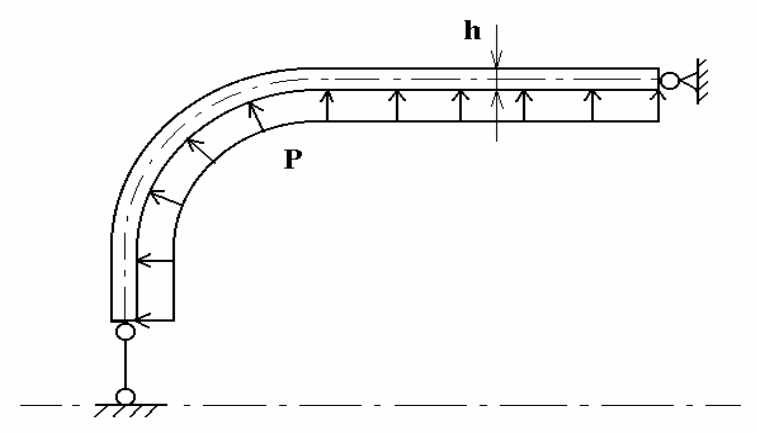

Fig. 2. Apparatus casing.

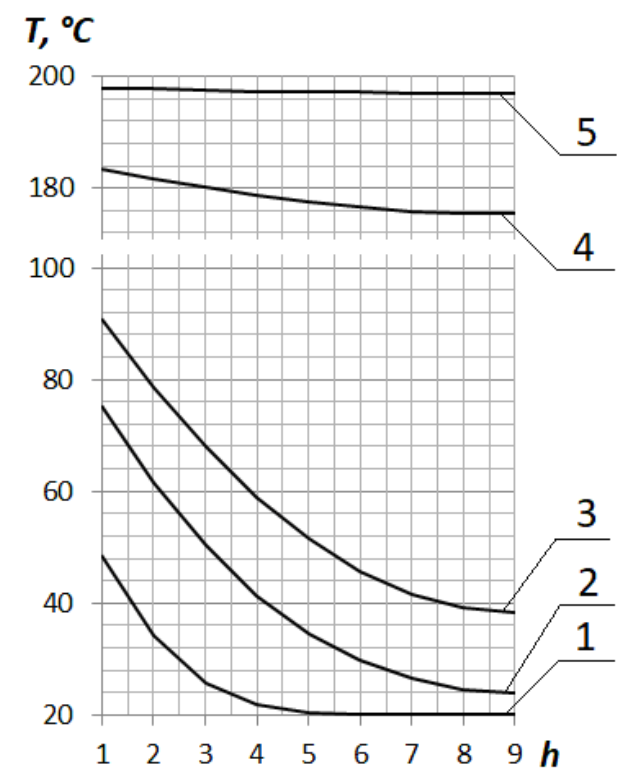

Fig. 3. Temperature distribution over the wall thickness of the diffusion apparatus. 


\section{C, ppm}

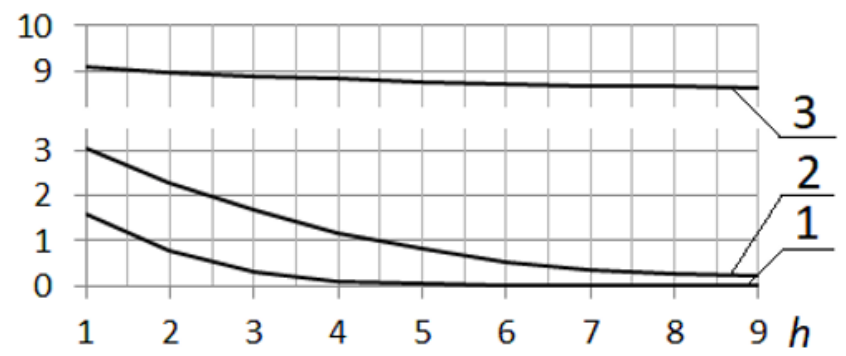

Fig. 4. Hydrogen distribution over the wall thickness.

When the internal pressure is raised to $p=8 \mathrm{MPa}$, the problem becomes physically nonlinear. For example, at a pressure of $p=6 \mathrm{MPa}$, it takes 16 approximations to reach the required solution accuracy chosen as $1 \%$ for the adopted mechanical properties at a concentration of $c=1.68 \mathrm{ppm}$, and 10 approximations for $c=9.47 \mathrm{ppm}$ and $c=15.93 \mathrm{ppm}$. At the same time, plastic deformation zones appear in the shell.

At a pressure of $\mathrm{p}=8 \mathrm{MPa}, 44$ approximations are needed to reach the required accuracy for concentration $\mathrm{c}=1.68 \mathrm{ppm}, 67$ approximations for $\mathrm{c}=9.47 \mathrm{ppm}$, and 69 approximations for $\mathrm{c}=15.93 \mathrm{ppm}$.

There are various approaches to estimating the strength of the shell structures. It appears that the simplest estimation of the strength of a shell structure can be obtained from the intensity of tangential stresses G (11), in the most loaded structure point comparing it with $\mathrm{G}$ (10). Another deformation criterion for the structure's lost load bearing capacity can be the intensity of shear deformations in $\mathrm{H}(11)$, which should be compared with intensity $\mathrm{H}$ (10). Increasing the pressure above $\mathrm{p}=8 \mathrm{MPa}$ leads to shell failure. Fig. 5 shows the variation in intensity $G$ with increasing pressure $p$ in the most loaded shell point at the inner surface. Curves 1, 2, 3 correspond to the calculations for the adopted mechanical properties at concentrations of $\mathrm{c}=1.68 \mathrm{ppm}, \mathrm{c}=9.47 \mathrm{ppm}, \mathrm{c}=15.93 \mathrm{ppm}$.

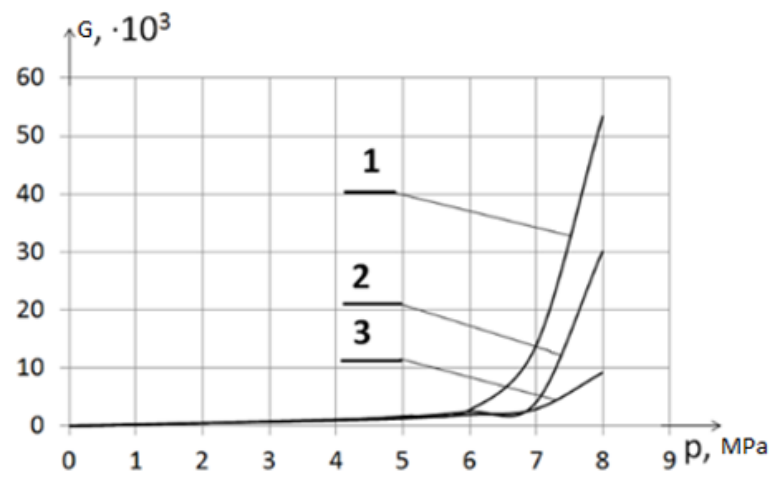

Fig. 5. Distribution of intensity $G$ with increasing pressure $p$ at the shell inner surface,

Fig. 6 shows the variation in intensity $\mathrm{G}$ with increasing pressure $\mathrm{p}$ in the most loaded shell point at the outer surface. Curves 1,2, 3 correspond to the calculations for the adopted mechanical properties at concentrations of $\mathrm{c}=1.68 \mathrm{ppm}, \mathrm{c}=9.47 \mathrm{ppm}, \mathrm{c}=15.93 \mathrm{ppm}$.

From Figs. 5 and 6 it can be inferred that beyond the pressure of $p=7 \mathrm{MPa}$ strong nonlinear effects of the stress-strain state that depends on hydrogen concentration come into play. 


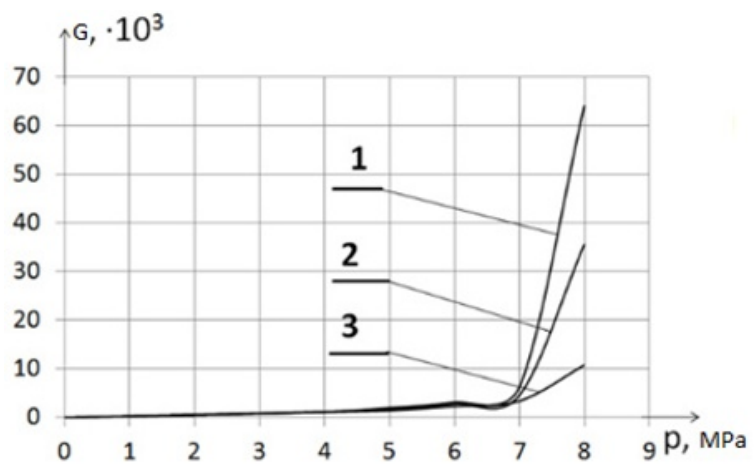

Fig. 6. Distribution of intensity $G$ with increasing pressure $p$ at the shell outer surface.

\section{Conclusion}

Therefore, using modern numerical methods and experimental data, the thermodiffusion problem of determination of the stress state of a thin-walled shell structure in the conditions of thermomechanical loading and exposure to aggressive environment has been solved. The subject of the study was a compound shell of revolution, the casing of a diffusion apparatus, loaded by internal pressure and operated in a hydrogen-containing environment at elevated temperatures. Generally, the problem of determination of the stress state of this thin-walled structure under given operating conditions is nonstationary and coupled. For the processes taking place at different physical time scales, the problem was presented as a sequence of separate boundary problems of heat propagation, hydrogen diffusion, and stress state calculation. Effective stresses and their invariants were determined by solving a physically nonlinear boundary problem. It was demonstrated that when the impact of an aggressive environment on the mechanical properties of metals is taken into account, the stress-strain state, and, as a consequence, the safety margin of structure elements changes.

\section{References}

1. D. Chapelle, Optimal Design of a Type 3 Hydrogen Vessel: Parti-Analytic Modeling of the Cylindrical Section. Internat. J. of Hydrogen Energy, no. 31. pp. 627-638 (2006)

2. Y. Inaba, T. Nishinara, Y. Nitta Analytical Study on Fire and Explosion Accidents Assumed in HTGR Hydrogen Production System. Nuclear Technology, 146, no. 1, pp. 49-57 (2004)

3. I.I. Ovchinnikov, I.G. Ovchinnikov, Impact of a Hydrogen-Containing Environment at Elevated Temperatures and Pressures on the Behavior of Metals and Structures Made of Them. "Science Studies" online magazine, no. 14. (In Russian) (2012)

4. A.A. Lakhdari, A. Seddak, I.I. Ovchinnikov, I.G. Ovchinnikov, Modeling Hydrogen Embrittlement of a Pipeline as a Thin-Walled Cylindrical Shell of Nonlinearly Elastic Material. "Scie. Studies" online magazine, 9, no. 4, (In Russian) (2017)

5. Yu.N. Rebyakov, A.O. Chernyavskiy, O.F. Chernyavskiy, Deformation and Failure of Materials and Structures under Diffusion. Bulletin of YuUrGU, no. 10, pp. 4-16. (In Russian) (2010)

6. A. Kh. Vorobiev, Diffusion Problems in Chemical Kinetics. Study guide. Moscow, Mosk. Univ., p.98 (In Russian) (2003) 
7. Yu.P. Cherdantsev, I.P. Chernov, Yu.I. Tyurin, Methods for Studying Metal-Hydrogen Systems: Study guide. Tomsk, ITPU, p. 286 (In Russian) (2008)

8. Ya.M. Grigorenko, A.T. Vasilenko, I.G. Emelyanov, et al.: Statics of Structural Elements. Kiev, A.S.K., p. 379, Mechanics of Composites: in 12 volumes, 8. (In Russian) (1999)

9. I.G. Emelyanov, V.I. Mironov, The Method for Estimation of Shell Hydrogenation with Variable Geometrical and Physics-Mechanical Parameters. AIP Conf. Proc. 2053, 030012; (2018) doi: 10.1063/1.5084373 